\title{
Creep in reactive colloidal gels: A nanomechanical study of cement hydrates
}

\author{
Michael Haist, ${ }^{1,2, *}$ Thibaut Divoux $\odot,{ }^{3,4, \dagger}$ Konrad J. Krakowiak, ${ }^{5}$ Jørgen Skibsted $\odot,{ }^{6}$ Roland J.-M. Pellenq, ${ }^{3,7}$ \\ Harald S. Müller, ${ }^{8}$ and Franz-Josef Ulm $\oplus^{2}$ \\ ${ }^{1}$ Institute for Building Materials, Leibniz University Hannover, 30167 Hannover, Germany \\ ${ }^{2}$ Department of Civil and Environmental Engineering, Massachusetts Institute of Technology, 77 Massachusetts Avenue, Cambridge, \\ Massachusetts 02139, USA \\ ${ }^{3}$ Department of Civil and Environmental Engineering, MultiScale Material Science for Energy and Environment, \\ UMI 3466, CNRS-MIT, 77 Massachusetts Avenue, \\ Cambridge, Massachusetts 02139, USA \\ ${ }^{4}$ Laboratoire de Physique, CNRS, University of Lyon, École Normale Supérieure de Lyon, \\ Université Claude Bernard, F-69342 Lyon, France \\ ${ }^{5}$ Civil and Environmental Engineering Department, Cullen College of Engineering, University of Houston, \\ 4726 Calhoun Road, Houston, Texas 77204-4003, USA \\ ${ }^{6}$ Laboratory for Solid-State NMR Spectroscopy of Inorganic Materials, Interdisciplinary Nanoscience Center (iNANO), \\ and Department of Chemistry, Aarhus University, DK-8000 C Aarhus, Denmark \\ ${ }^{7}$ EpiDaPo Lab - CNRS / George Washington University, Children's National Medical Center, Children's Research Institute, \\ 111 Michigan Avenue NW, Washington, DC 20010, USA \\ ${ }^{8}$ Institute for Concrete Structures and Building Materials, Karlsruhe Institute of Technology, Karlsruhe, Germany
}

(Received 6 December 2020; accepted 12 October 2021; published 19 November 2021)

\begin{abstract}
From soft polymeric gels to hardened cement paste, amorphous solids under constant load exhibit a pronounced time-dependent deformation called creep. The microscopic mechanism of such a phenomenon is poorly understood in amorphous materials and constitutes an even greater challenge in densely packed and chemically reactive granular systems. Both features are prominently present in hydrating cement pastes composed of calcium silicate hydrate (C-S-H) nanoparticles, whose packing density increases as a function of time, while cement hydration is taking place. Performing nanoindentation tests and porosity measurements on a large collection of samples at various stages of hydration, we show that the creep response of hydrating cement paste is mainly controlled by the interparticle distance and results from slippage between $(\mathrm{C}-\mathrm{S}-\mathrm{H})$ nanoparticles. Our findings provide a unique insight into the microscopic mechanism underpinning the creep response in aging granular materials, thus paving the way for the design of concrete with improved creep resistance.
\end{abstract}

DOI: 10.1103/PhysRevResearch.3.043127

\section{INTRODUCTION}

Under constant external load, solid materials display an instantaneous elastic strain followed by pronounced timedependent deformations. Such a mechanical response, called creep, is ubiquitously observed in both crystalline (i.e., ordered) and amorphous (i.e., disordered) materials, such as gels, glasses, granular systems, and hardened cement paste [1-3]. While the creep behavior of crystalline materials has been quantitatively associated with the collective motion of dislocations in such materials [4-8], the microscopic mechanism underpinning the creep response in amorphous granular materials remains controversial and a topic of intense research [9-13].

\footnotetext{
*michael.haist@baustoff.uni-hannover.de

†thibaut.divoux@ens-lyon.fr
}

Published by the American Physical Society under the terms of the Creative Commons Attribution 4.0 International license. Further distribution of this work must maintain attribution to the author(s) and the published article's title, journal citation, and DOI.
Indeed, in granular matter the creep response is highly sensitive to the particle volume fraction as well as the interparticle interactions. On the one hand, in colloidal gels, i.e., systems composed of attractive particles forming a percolated network at low volume fraction, the early-stage creep response is often accounted for by linear viscoelastic properties, i.e., macroscopically reversible deformations, up to a critical strain value beyond which a sudden burst of localized plastic rearrangements triggers the nucleation of microscopic cracks long before the macroscopic failure of the material $[11,14,15]$. On the other hand, in densely packed granular materials, creep is often ascribed to a disturbance of the force network present in the material microstructure, which corresponds to plastic rearrangements [16,17]. In the limit of jammed systems, which lack sufficient free volume for local reorganization of the granular network, the detailed mechanisms of creep are not fully understood. Creep here is often associated with a dilation of the particle system [18,19], and for large stresses, the individual deformation of the particles and their fracture has been observed to strongly enhance the creep process [20,21].

Identifying and modeling the microscopic mechanism of the creep response in amorphous granular materials is further 
complicated by the presence of chemical reactions such as dissolution, precipitation, and chemical bonding, which result in irreversible aging and a pronounced morphological evolution of the material's microstructure [22-25]. The interplay between the changes induced by these reactions and those triggered by the external loading make the creep response at the microscopic scale much more complex to decipher. This is the case for hardened cement paste, which is the "glue" that provides strength to concrete [26]. The reactive nature of this system and its time-evolving microstructure pose a significant challenge for studying its creep behavior and, at the same time, offer a unique opportunity for determining the impact of packing density on the creep response of granular materials in general.

The mechanical properties of hardened cement paste are governed by colloidal particles of calcium silicate hydrate $(\mathrm{C}-\mathrm{S}-\mathrm{H})$, which precipitate as nanoparticles after mixing and dissolution of the polydisperse cement powder in water $[27,28]$. These colloidal particles interact via short-range attraction and longer-range electrostatic repulsion forces, which are strongly influenced by the ion content and composition of the solvent (with ions resulting from the cement dissolution) [29-33]. Eventually, hardened cement paste forms a complex nanoporous network of colloidal particles, which is age and composition dependent. In consequence, depending on the degree of hydration $\xi$ (defined as the fraction of cement powder having reacted with water at a certain point in time), the water-to-cement mass ratio $w / c$, the age of the sample when the mechanical load is applied, and the moisture conditions, hardened cement paste shows a broad range of creep responses under a constant external load [34-37].

To date, three different microscopic mechanisms have been proposed to account for the macroscopic reversible and irreversible creep response of hardened cement paste. The underlying concepts, however, can be generalized and adapted to basically any kind of granular system. These scenarios explain the creep behavior either as a shear deformation of the individual C-S-H colloidal particles (length scale of approximately $4 \mathrm{~nm}$ ) [38], as a slippage between these particles (length scale of approximately up to $20 \mathrm{~nm}$ ) [37], or as the formation of microcracks at spatial scales significantly larger than the particle size [39]. Despite substantial evidence for the existence of all three of these mechanisms [40-43], their respective contribution to the macroscopic creep response is unknown. Moreover, these static scenarios take into account neither the reactive and time-dependent nature of cement paste nor its heterogeneous microstructure. Indeed, hardened cement paste is not homogeneous on a microstructural level, but is primarily formed by the precipitation of C-S-H particles, which, however, prevail in regions of low and high (packing) density. These regions are often referred to as LD-C-S-H and HD-C-S-H, respectively [44-46]. Their effect on the creep behavior and creep mechanisms so far has not been considered. Finally, insight into the different creep mechanisms on the submicrometer scale and nanoscale has only recently been gained using numerical simulations [47,48]. Whereas Ref. [47] reports clear evidence for creep deformation of individual particles, Ref. [48] showed that the main contributing factor to creep must be seen in a slippage between particles.
Unfortunately, substantial experimental evidence to test either of these numerical results is still missing.

Building upon the statistical analysis of an extensive series of nanoindentation tests on cement paste samples that had reached different degrees of hydration, we provide a local scenario for the creep response of hardened cement paste that takes into account the heterogeneous and time-evolving properties of cement's microstructure. Spatially resolved mechanical properties are coupled to elemental chemistry surface mapping to isolate the mechanical properties of the so-called LD- and HD-C-S-H at the submicrometer level. As a key result, we show that for both types of C-S-H phases, the major contribution to the creep response is set by the slippage between $\mathrm{C}-\mathrm{S}-\mathrm{H}$ nanoparticles. Our results show that LD- and HD-C-S-H phases only differ by their respective volume fraction in $\mathrm{C}-\mathrm{S}-\mathrm{H}$ particles and thus behave as a single granular phase when it comes to the creep response. In that framework, the creep response of hardened cement paste is mainly controlled by the interparticle distance, which decreases for increasing degree of hydration as more C-S-H particles precipitate. Finally, we further confirm this picture by porosimetry measurements, which allow us to quantify the porosity of the cement paste as a function of the degree of hydration. Such information about the porosity is essential to connect the findings on hardened cement paste to the creep behavior of densely packed and possibly jammed granular systems in general.

The outline of this paper is as follows: Sec. II provides an overview regarding cement hydration in terms of chemical reaction and describes the emergence of hardened cement paste microtexture. Section III recalls the state of the art regarding key factors controlling the mechanical properties of hardened cement paste. We then present our experimental results in Sec. IV, where we report the submicrometer creep response of the paste as a function of the packing density of the C-S-H particles, as well as microstructural measurements by nuclear magnetic resonance (NMR) spectroscopy and porosimetry. Finally, we summarize our main conclusions in Sec. V.

\section{OVERVIEW ON CEMENT HYDRATION}

Hardened cement paste (HCP) forms during the reaction of ordinary Portland cement-primarily consisting of calciumsilicate phases $3 \mathrm{CaO} \cdot \mathrm{SiO}_{2}$ and $2 \mathrm{CaO} \cdot \mathrm{SiO}_{2}$ (abbreviated $\mathrm{C}_{3} \mathrm{~S}$ and $\mathrm{C}_{2} \mathrm{~S}$, respectively) and minor fractions of calciumaluminate phase $3 \mathrm{CaO} \cdot \mathrm{Al}_{2} \mathrm{O}_{3}\left(\mathrm{C}_{3} \mathrm{~A}\right)$ and $\mathrm{CaSO}_{4} \cdot 2 \mathrm{H}_{2} \mathrm{O}$ -with water. In the rest of this paper, the oxide phases in cement chemistry are abbreviated as follows: $\mathrm{C}$ for $\mathrm{CaO}, \mathrm{S}$ for $\mathrm{SiO}_{2}, \mathrm{H}$ for $\mathrm{H}_{2} \mathrm{O}$, and $\mathrm{A}$ for $\mathrm{Al}_{2} \mathrm{O}_{3}$.

From the hydration of calcium silicates, two products form via a dissolution-precipitation reaction: amorphous calcium silicate hydrate $(\mathrm{C}-\mathrm{S}-\mathrm{H})$ and crystalline calcium hydroxide $(\mathrm{CH})$, with the amount of $\mathrm{C}-\mathrm{S}-\mathrm{H}$ being formed clearly outweighing the $\mathrm{CH}$ phase [28]. Moreover, C-S-H forms via precipitation at a density of about $2.45 \mathrm{~g} / \mathrm{cm}^{3}$, a mean $\mathrm{CaO}$-to- $\mathrm{SiO}_{2}$ ratio (or $\mathrm{C} / \mathrm{S}$ ) of 1.7 , forming bricklike particles with a typical edge length of approximately $4.2 \mathrm{~nm}$ [Fig. 1(e)] that show a layered microstructure consisting of calcium silicate hydrate and water [27,49]. These particles randomly aggregate to form larger structures, incorporating 
degree of hydration $\xi=0$ (immediatly after water addition)

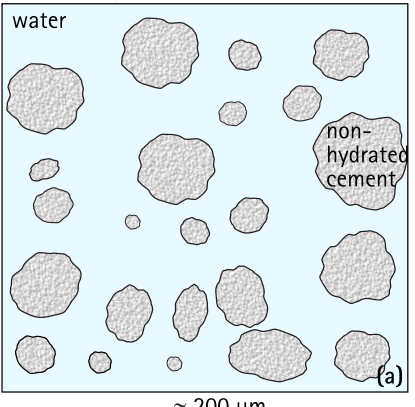

degree of hydration $\xi \approx 0.6$
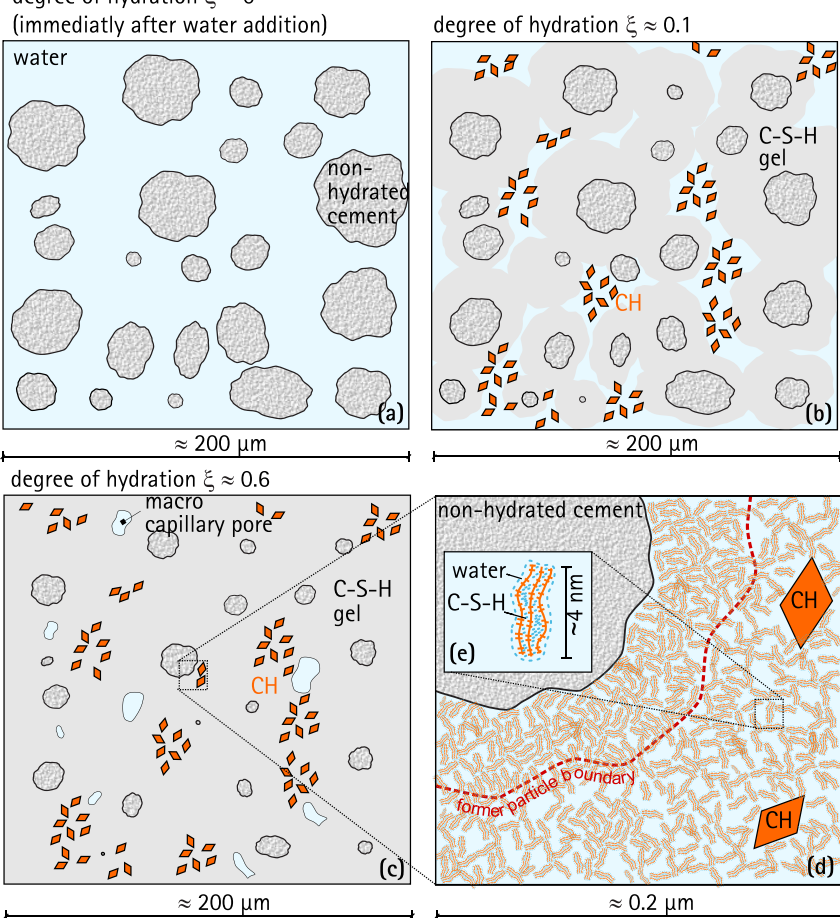

FIG. 1. Sketch depicting the hydration process of cement particles in water (a), which results in the formation of hardened cement paste consisting of nonhydrated cement particles, C-S-H phases, $\mathrm{CH}$ phases, and pores of different size and content [(b) and (c)]; due to the hydration process, cement particles dissolve, and $\mathrm{C}-\mathrm{S}-\mathrm{H}$ and $\mathrm{CH}$ precipitate in the solvent in a less densely packed manner outside the (former) particle surface and in a tighter manner within the bounds of the former particle surface (which was dissolved) (d). Gel pores designate voids between the $\mathrm{C}-\mathrm{S}-\mathrm{H}$ particles; capillary pores designate voids formerly occupied by the solvent. (e) Zoom area in (d) of a bricklike C-S-H particle.

more or less water, thus showing significant differences in packing density $\eta$ and density as sketched in Fig. 1(d). The water incorporated inside as well as between the particles is referred to as gel water, which occupies the so-called gel porosity, with pores of up to $30 \mathrm{~nm}$ in diameter [Figs. 1(d) and $1(\mathrm{e})]$.

Whereas $\mathrm{CH}$ primarily precipitates in the bulk of the solvent as platelike crystals with a typical size reaching up to several micrometers [Figs. 1(a)-1(d)], C-S-H particles nucleate on the surface of the dissolving cement particle, thus creating a shell of hydration products around the latter, and in turn, increasingly hinder the dissolution process of the cement [Fig. 1(d)] [50]. Due to this shell-like growth mechanism, seminal works on cement hydration started differentiating between two types of C-S-H, namely, (i) C-S-H forming outside of this shell in the free space between cement particles yielding a loose-packing assembly of C-S-H particles, thus termed the outer product or low-density C-S-H (LD-C-S-H), and (ii) $\mathrm{C}-\mathrm{S}-\mathrm{H}$ forming within the shell and thus the space cleared by the progressing dissolution of the cement, showing a significantly higher packing density and thus being termed the inner product or high-density C-S-H (HD-C-S-H) [44]. LD- and HD-C-S-H are chemically quasi-identical with respect to their solid constituents. However, they possess a different packing density, porosity, and density, on the basis of which they can be clearly distinguished. Yet, the packing-density distributions of LD- and HD-CSH show a pronounced overlap.

Originally, it was believed by Jennings and others (see, e.g., Ref. [27]) that the mentioned properties such as packing density are imminent to the phases; however, in this paper we can clearly show that they evolve in time as a function of hydration. Both LD- and HD-C-S-H as well as $\mathrm{CH}$ (in the following termed as cement gel) form in the space between the cement particles [Figs. 1(a)-1(d)]. By chemically and physically binding water, the volume of $\mathrm{C}-\mathrm{S}-\mathrm{H}$ and $\mathrm{CH}$ formed during this hydration process is approximately 2.13 times greater than the volume of the hydrated cement [51]. In terms of microtexture, the hydration process thus consists in filling up the space between the cement particles (originally filled with water) with a mix of C-S-H gel (more or less densely packed C-S-H particles) and $\mathrm{CH}$. In the early stage of hydration, hardened cement paste is highly porous, and the corresponding voids are referred to as capillary pores, with a diameter up to several micrometers. With continuing hydration, the capillary pore space is filled with $\mathrm{C}-\mathrm{S}-\mathrm{H}$ gel and $\mathrm{CH}$, leading to a densification of the system. For pastes with high water content (and thus relatively low cement content), the amount of gel formed during hydration does not suffice to fill the entire space. In practice, for a water-to-cement mass ratio smaller than 0.4 , large capillary pores remain in the paste even when the entire cement powder has reacted [51]. In conclusion, gel pores form due to the chemical reaction process, whereas capillary pores are remnants of the interstitial space between the original cement particles.

\section{MECHANICAL PROPERTIES OF HARDENED CEMENT PASTE}

The macroscopic mechanical properties of HCP are primarily governed by the capillary porosity and the degree of hydration $\xi$, i.e., the share of cement, which has already dissolved to form C-S-H gel and $\mathrm{CH}$. With increasing $\xi$ and thus decreasing capillary porosity, the strength of HCP increases, and the creep rate (i.e., the change in creep deformation over time) decreases [51,52]. Nonetheless, even a fully hydrated HCP with $\xi=1.0$ with no capillary pores [i.e., a water-tocement ratio $(w / c)$ lower than 0.4$]$ shows pronounced creep. Thus a closer look has to be given to the mechanical properties of the gel's constituents and their corresponding mechanical interaction behavior. Moreover, HCP is a highly porous system with pore sizes ranging from fractions of a nanometer to micrometers; hence the mechanical properties at all scale levels are also strongly influenced by the water content of the system. Gel pores are normally fully filled with water, whereas water in the capillary pores is predominantly present in the form of water films [51,53,54]. In consequence, depending on the degree of hydration, the water-to-cement mass ratio $w / c$, and the moisture conditions, HCP shows only minor creep for dry conditions at very low $w / c$ and very high degree of hydration, and very substantial creep in the opposite case when subjected to a constant external load [34-36].

Concerning the submicrometer mechanical properties of the cement gel, molecular dynamics simulations on individual 
$\mathrm{C}-\mathrm{S}-\mathrm{H}$ particles have shown that clear trends between the $\mathrm{Ca}-$ to-Si ratio of the particles and the submicrometer mechanical properties can be established [55]. For a typical Ca-to-Si ratio as observed in C-S-H gel from Portland cement hydration, the stiffness $\mathrm{M}$ of the individual particles was determined to be about $72 \mathrm{GPa}$. Investigations regarding the interaction of the individual $\mathrm{C}-\mathrm{S}-\mathrm{H}$ particles revealed that the latter interact via short-range attraction and longer-range electrostatic repulsion forces, which are strongly influenced by the ion content and composition of the pore water [31]. Furthermore, significant efforts in simulating these colloidal interactions allowed for an accurate modeling of the C-S-H gels' physical (and mechanical) properties [29,30,32,33,48]. In particular, these simulations closely reproduce results obtained from experimental nanoindentation on HCP $[46,55]$.

\section{RESULTS}

In order to determine the creep response of hardened cement paste at different stages of the hydration process, we have prepared a series of 120 samples, whose hydration process is stopped at 30 different points in time by solvent exchange with isopropanol (see Appendix A for details) [56]. These samples were subjected to various investigation techniques such as statistical nanoindentation (see Appendix B), chemical surface mapping (see Appendix C), and various bulk measurement techniques (see Appendix D). The sample preparation process, conducted over a year, yielded a series of samples with various hydration degrees $\xi_{\mathrm{NMR}}$ for the cement silicate phases (i.e., alite and belite) ranging from 0.2 to 0.7 as determined by ${ }^{29} \mathrm{Si}$ NMR spectroscopy (see Appendix D for details). Note that this NMR method is primarily focused on the hydration behavior of the calcium-silicate phases and neglects the contribution of calcium-aluminate phases to hydration. To monitor the evolution of the cement paste's mechanical properties at the microscale for increasing degree of hydration, we have conducted nanoindentation tests to determine the hardness $H$, the indentation modulus $M$, and the creep modulus $C$ at a scale of approximately $1 \mu \mathrm{m}^{3}$.

The indentation hardness $H$ is defined as the ratio of the maximum force $P_{\max }$ applied by an indenter onto the sample, and the projected contact area $A_{c}$ between the intender and the indented surface [57]. The hardness $H$ correlates to the strength of the sample. The indentation modulus $M$ relates to the elasticity of the indented material and is defined as $M=E /\left(1-v^{2}\right)$, where $E$ is Young's modulus and $v$ is the Poisson ratio of the sample. In practice, $M$ is determined following the method of Oliver and Pharr by measuring the slope $S=d P /\left.d h\right|_{h=h_{\max }}$ during the unloading step of an indentation experiment and computing $M=S /\left(2 a_{u}\right)$, where $a_{u}=\sqrt{A_{c} \pi}$ is the radius of contact between the indenter probe and the indented surface upon unloading $[57,58]$. Finally, the indentation creep modulus $C$ is derived from the contact creep compliance $L(t)$, which verifies $L(t)=$ $2 a_{u} \Delta h(t) / P_{\max }+$ const, for short experiments, i.e., limited to a few minutes [59]. For a logarithmic time dependence of the creep phenomenon, i.e., $\Delta h(t) \sim x_{1} \ln (1+t / \tau)$ as observed here, the rate of creep compliance reads $d L(t) / d t=1 /(C t)$, with $C=P_{\max } /\left(2 a_{u} x_{1}\right)$. In that framework, the parameter $C$ has the unit of a stress as well, but, as the load $P_{\max }$ is constant in the previous equation, $C$ is quasiproportional to the inverse creep rate, i.e., the (inverse) velocity of the creep process. For more details on the experimental determination of $H, M$, and $C$, see Appendix B.

In the following, we restrain the data analysis to continuous force-depth data, whereas outliers including curves with discontinuities such as jumps, etc., are not considered (see Sec. IV D for the analysis of force-depth data with jumps). A sample is typically characterized by a nanoindentation grid of $25 \times 25$ indentations yielding 625 triplets $(H, M, C)$, which are statistically analyzed with a Gaussian mixture modeling approach (see Appendix B for details and Ref. [60]). This procedure allowed us to identify up to five different phases, i.e., two types of $\mathrm{C}-\mathrm{S}-\mathrm{H}$ phases, a $\mathrm{CH}$ phase, nonreacted cement particles, and a mixed phase consisting of C-S-H and $\mathrm{CH}$ [61].

We illustrate the relevance of the clustering method to capture the properties of the individual phases composing HCP on a sample with a degree of hydration $\xi_{\mathrm{NMR}}=0.6$. A picture obtained by electronic microscopy of a region of interest on the sample surface is shown in Fig. 2(a). The corresponding spatial distribution of the $\mathrm{CaO}-\mathrm{to}-\mathrm{SiO}_{2}$ ratio obtained via elemental chemical mapping is shown in Fig. 2(b) (for details on the elemental chemical mapping, see Appendix C), while that of the indentation modulus $M$ obtained by nanoindentation is shown in Fig. 2(c). The nonhydrated cement grains visible as light gray areas on the SEM image are clearly visible in both the chemical and mechanical maps. These grains correspond to the areas with the highest value of $M$ in Fig. 2(c) outweighing that of the hydration product surrounding the particles and to a $\mathrm{CaO}$-to- $\mathrm{SiO}_{2}$ ratio ranging between 2.0 and 3.5 in Fig. 2(b), reflecting the dominant effect of the mineral tricalcium silicate $\left(\mathrm{CaO} / \mathrm{SiO}_{2}=3.0\right)$ making up the cement. Some remnants of cement grains consisting purely of dicalcium silicate with $\mathrm{CaO} / \mathrm{SiO}_{2}=2.0$ are also visible in Fig. 2(b); see, e.g., coordinates $X=150 \mu \mathrm{m}, Y=50 \mu \mathrm{m}$ ), and their indentation modulus also significantly exceeds that of the reaction products [Fig. 2(c)]. Due to their significantly higher indentation modulus $M$ (and hardness $H$; not shown) the cement particles are correctly identified by the Gaussian mixture algorithm as individual clusters.

The map corresponding to the $\mathrm{CaO}$-to- $\mathrm{SiO}_{2}$ ratio further shows clusters with very high $\mathrm{CaO}-$ to- $\mathrm{SiO}_{2}$ ratios [see red color in Fig. 2(b)], hinting to patches dominated by $\mathrm{CH}$. These patches display a channel-like distribution, reflecting their formation history, i.e., $\mathrm{CH}$ precipitating in the free water between the cement particles. Looking at the mechanical map [Fig. 2(c)], the $\mathrm{CH}$ patches only lead to a minor decrease in elasticity, due to the fact that the indentation modulus of pure $\mathrm{CH}$ is insignificantly higher (approximately a factor of 1.5) than that of C-S-H. The Gaussian mixture modeling approach identifies these patches as individual clusters.

Finally, we observe in Fig. 2(b) that the space between the cement particles and the $\mathrm{CH}$ channels is filled with a mineral phase with a $\mathrm{CaO}-$ to- $\mathrm{SiO}_{2}$ ratio between 1.5 and approximately 2.5 , which is identified as $\mathrm{C}-\mathrm{S}-\mathrm{H}$. The mechanical properties of this phase determined by indentation further allow the distinction of two distinct subphases, their mechanical properties corresponding to the mechanical 

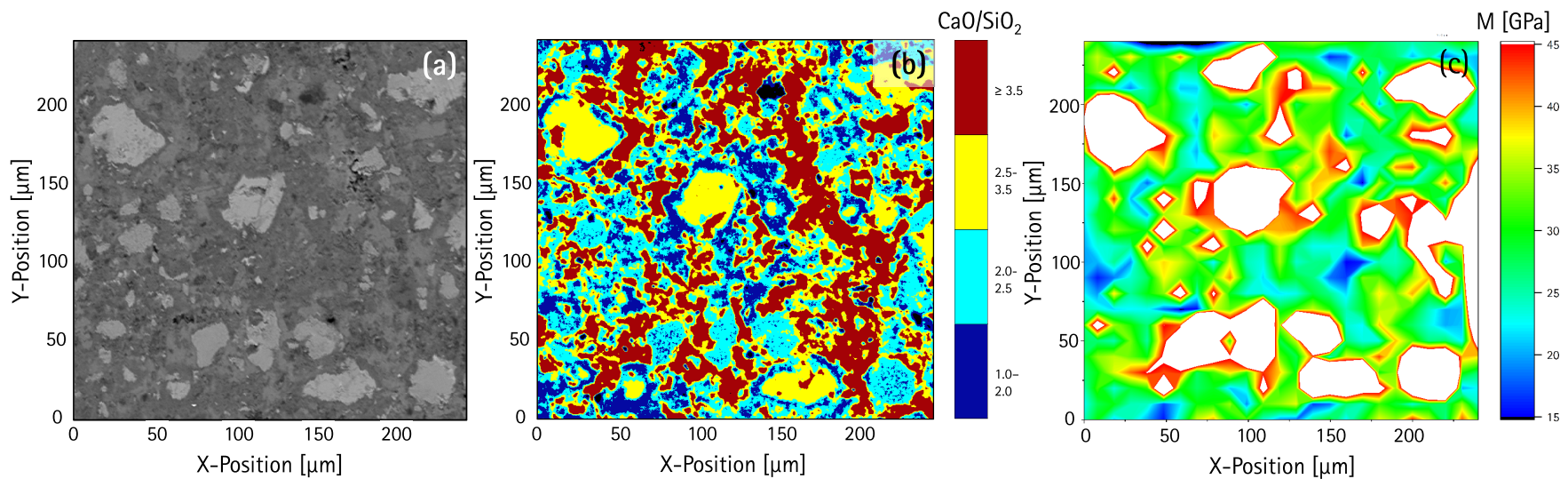

FIG. 2. (a) Scanning electron microscope (SEM) backscattered image of the surface of a sample of hardened cement paste. Spatial distributions: (b) map of the $\mathrm{CaO}$-to- $\mathrm{SiO}_{2}$ ratio determined via wavelength-dispersive $\mathrm{x}$-ray spectroscopy (WDS) element mapping of Ca and $\mathrm{Si}$ and converted into an oxide ratio, and (c) map of the indentation modulus $M$ determined by nanoindentation. Data were measured on a cement sample with a degree of hydration $\xi_{\mathrm{NMR}}=0.6$.

properties of LD- and HD-C-S-H as identified in previous research [60].

\section{A. Impact of cement hydration on the submicrometer mechanical response of hardened cement paste}

Figure 3 shows an overview of the entirety of the mechanical results for the two phases with the lowest mechanical properties as identified by the Gaussian mixture algorithm. Following the reasoning outlined with Fig. 2, these two phases can be classified as LD- and HD-C-S-H, and their mechanical properties are in agreement with those of the literature $[3,62]$. The composition of the samples is dominated by these two phases [Fig. 3(d)], making up for more than $75 \%$ of the hydration products, with the individual contents, however, being subject to increased scatter. The volume fraction of LD-C$\mathrm{S}-\mathrm{H}$ increases with the degree of hydration $\xi_{\mathrm{NMR}}$, especially for $\xi_{\mathrm{NMR}} \gtrsim 0.5$, whereas the content of HD-C-S-H remains constant at a volume fraction of about $35 \%$ over the entire range $0.2 \lesssim \xi_{\mathrm{NMR}} \lesssim 1$. Remarkably, both LD- and HD-C-S-H show increasing values of $H, M$, and $C$ for increasing degree of hydration $\xi_{\text {NMR }}$ [Figs. 3(a)-3(c)]. Note that these results are robust, whether the phase determination is obtained by clustering the results solely based on the mechanical properties $(H, M$, and $C)$ or by additionally taking into account the chemical (elemental) composition in $\mathrm{Ca}, \mathrm{Si}$, and $\mathrm{Al}$ determined at the location of the indents by wavelength-dispersive $\mathrm{x}$-ray spectroscopy [see half-filled symbols in Figs. 3(a)-3(d)]. See also Appendix C for more details.

The LD- and HD-C-S-H phases can be modeled as an assembly of C-S-H nanoparticles interacting by cohesive forces [27]. Using a micromechanical approach introduced in Refs. $[63,64]$ and assuming the C-S-H particle stiffness to be $72 \mathrm{GPa}$ [55], we determine the packing density $\eta$ of each phase as a function of the degree of hydration [Fig. 3(h)]. This determination was based on the solution of the inverse problem, formulated within the framework of cohesive granular materials, in which a least-squares minimization was employed to determine the packing density $\eta$, as well as particles' intrinsic properties [63]. For both LD- and HD-C-S-H phases, $\eta$ increases with $\xi_{\text {NMR }}$ [Fig. 3(h)]. The difference in packing density $\Delta \eta$ between the two phases remains constant, equal to approximately 0.05 , for degrees of hydration up to $\xi_{\mathrm{NMR}} \simeq 0.55$, before decreasing and vanishing in the limit of complete hydration $\left(\xi_{\mathrm{NMR}}=1\right)$. This result supports the picture that the formation of new LD-C-S-H phases in the larger capillary pore space of hydrating cement paste is significantly influenced by the free volume available for this precipitation process to occur, leading to more densely packed phases at higher degrees of hydration. Moreover, the formation of new LD-C-S-H for increasing degrees of hydration goes along with a continuous compaction of the existing LD-C-S-H phases, shifting their classification from LD- to HD-C-S-H together with a compaction of the existing HD-C-S-H phases. The terminal packing density $\eta_{\max }$ is limited to values of about 0.75 [Fig. 3(h)], in good agreement with the maximum packing density of monosized spheres [49]. In that framework, the mechanical properties of LD- and HD-C-S-H phases can now be plotted as a function of the packing density $\eta$ of C-S-H particles [Figs. 3(e)-3(g)]. $H, M$, and $C$ grow linearly (at least in the considered range) for increasing packing density, and no distinction can be made between the results of the LDand HD-C-S-H phases. This result is remarkable and shows that the packing of the individual C-S-H nanoparticles is the fundamental parameter determining the mechanical properties of both LD- and HD-C-S-H phases. Moreover, this result suggests there is a continuum between these two phases that only differ by their packing density.

Following the approach employed on mesoscale simulations [46], the packing density $\eta$ can be used to estimate the ratio between the average interparticle distance $r$ and the diameter $d$ of the C-S-H particles as follows: $r / d=\eta^{-1 / 3}$ [65]. As can be seen in Fig. 4(a), the creep modulus $C$ increases roughly linearly with decreasing interparticle distance $r / d$, over the range of packing densities that can be accessed experimentally. This trend corresponds to a significant reduction in creep rate and strongly suggests that the creep response of hardened cement paste primarily depends on interparticle slippage processes, and not on the creep behavior of the individual C-S-H particles. Hence early-age creep occurs at the intergranular scale and not at a spatial scale smaller than the size of an individual C-S-H particle. Moreover, Fig. 4(b) 

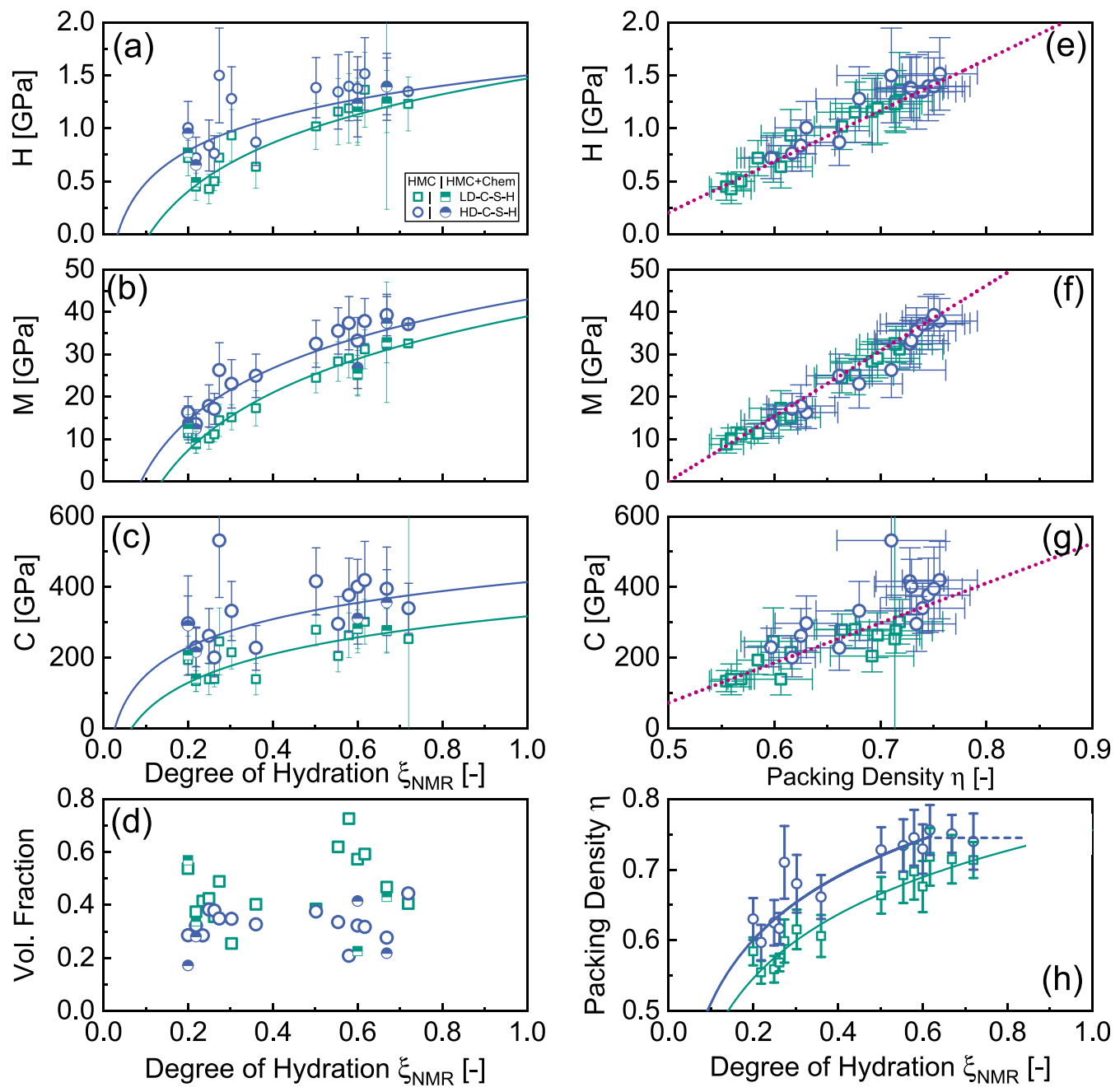

FIG. 3. Mechanical properties as a function of the degree of hydration. (a)-(c) Indentation hardness $H$, indentation modulus $M$, and creep modulus $C$ for LD-C-S-H and HD-C-S-H phases determined by statistical nanoindentation as a function of degree of hydration $\xi_{\mathrm{NMR}}$ determined by ${ }^{29} \mathrm{Si}$ NMR spectroscopy. Both phases are identified by a statistical analysis based on $H, M$, and $C$ (index HMC, open symbols) or based on $H, M$, and $C$ and the chemical composition in calcium, silicon, and aluminum at the locus of the indents (index HMC-chem, half-filled symbols) as input values. Solid lines are logarithmic functions, which serve as guides to the eye. (e)-(g) Same data, $H, M$, and $C$, as in (a)-(c) vs packing density $\eta$ computed by micromechanical modeling (see text) of C-S-H particles in the respective phases. Dotted lines correspond to the best linear fit of the data. (d) Volume (Vol.) fraction $f$ and (h) packing density $\eta$ of LD- and HD-C-S-H phases vs degree of hydration $\xi_{\mathrm{NMR}}$. The blue dashed line corresponds to a packing density of 0.74 , and solid lines serve as guides to the eye. In all the graphs, the error bars stand for twice the standard deviation of the considered observable.

shows that this process is valid for both LD- and HD-C-S-H, as both HD- and LD-C-S-H packing fraction distributions exhibit significant overlap. For $\xi_{\mathrm{NMR}} \geqslant 0.6$ the interparticle distance strives to an end value, indicating that the hydration process seems to be controlled by packing limitations for high degrees of hydration. Finally, the difference in interparticle distance between LD- and HD-C-S-H decreases for increasing degree of hydration [Fig. 4(c)]. Both results indicate that the system is entering a jammed state for high degrees of hydration, which significantly slows down creep, but does not prevent it totally.

\section{B. Creep response controlled by interparticle sliding friction}

Additional support for these findings is derived from ${ }^{29} \mathrm{Si}$ magic angle spinning (MAS) NMR experiments, which allow us to identify the different types of condensation of silicate tetrahedra in the $\mathrm{C}-\mathrm{S}-\mathrm{H}$ nanoparticles, i.e., dimers and silicate end groups (denoted $Q^{1}$ ), members of silicate chains $\left(Q^{2}\right)$, and silicate tetrahedra neighboring Al tetrahedra in the chain $\left[Q^{2}(1 \mathrm{Al})\right]$ [Fig. 5(a)]. Dimers form by precipitation processes in the supersaturated pore solution before polymerizing into $\mathrm{C}-\mathrm{S}-\mathrm{H}$ phases with larger chain lengths $Q^{2}$ and $Q^{2}(1 \mathrm{Al})$ [Fig. 5(b)]. This polymerization process is especially pronounced for low values of $\xi_{\mathrm{NMR}}$. However, while the formation rate of $\mathrm{C}-\mathrm{S}-\mathrm{H}$ particles with longer silicate chains seems to be independent of $\xi_{\text {NMR }}$ (compare the slope of the $Q^{2}$ curve for $0.1 \leqslant \xi_{\mathrm{NMR}} \leqslant 0.4$ and for $\xi_{\mathrm{NMR}} \geqslant 0.6$ ), the fraction of $Q^{1}$ sites decreases slightly for $0.25 \leqslant \xi_{\mathrm{NMR}} \leqslant 0.4$ but then undergoes a clear increase for $\xi_{\mathrm{NMR}} \simeq 0.5$. The small decrease in the fraction of $Q^{1}$ sites may reflect that dimers join by a silicate bridging tetrahedron, forming pentamers or longer 

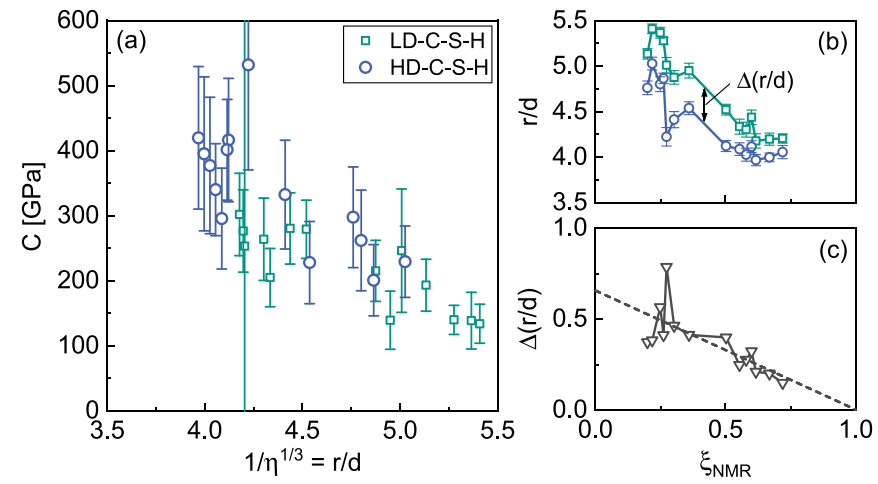

FIG. 4. (a) Creep modulus $C$ as a function of $r / d$, the ratio of the distance between C-S-H nanoparticles in LD- and HD-C-S-H phases normalized by the nanoparticle size. (b) Interparticle spacing $r / d$ for LD- and HD-C-S-H phases and (c) difference between LD- and HDC-S-H interparticle distance $\Delta(r / d)$ as a function of the degree of hydration $\xi_{\mathrm{NMR}}$. The dashed line in (c) corresponds to the best linear fit of the data.

chains. The subsequent increase in $Q^{1}$ sites is concomitant to the decrease in average $\mathrm{C}-\mathrm{S}-\mathrm{H}$ chain length (i.e., the average number of silicate tetrahedra, including tetrahedral $\mathrm{Al}$, in the chains), as seen in Fig. 5(b) and is in good agreement with previous results from studies of white Portland cement hydration [66]. This observation may reflect an increased incorporation of $\mathrm{Ca}^{2+}$ ions with respect to the number of silica tetrahedra in the interlayer of the C-S-H structure, which will split longer chain units into dimers in the $\mathrm{C}-\mathrm{S}-\mathrm{H}$, as observed when the Ca-to-Si ratio of the C-S-H phase increases.

However, despite a clear increase in average chain length (compared with the standard deviation of approximately 0.15 ) for low degrees of hydration $\xi_{\mathrm{NMR}} \leqslant 0.4$, no correlation between the chain length and the creep modulus $C$ can be observed [Fig. 5(c)], confirming that early-age creep occurs at the C-S-H interparticle scale and not at the subnanometer (molecular) scale as probed by solid-state NMR. This provides additional evidence that the creep behavior of the phases is not controlled by the internal structure or nanoscale properties of the individual C-S-H particles, but is rather happening at a larger spatial scale, for instance, by a sliding between particles. In this context, we emphasize that the drop in chain length observed for $\xi_{\mathrm{NMR}}$ values between approximately 0.38 and 0.50 goes along with substantial changes in interparticle spacing $r / d$ [see Fig. 4(b)]. This indicates that changes in the polymerization of the C-S-H do not influence the creep behavior directly and that these changes (i.e., polymerization) are related to the formation of new particles thus indirectly influencing the cement paste microstructure. The degree of hydration $\xi_{\mathrm{NMR}}$ at which the changes in fractions of $Q^{1}$ and $Q^{2}$ sites occur is the same degree of hydration beyond which there is a sudden increase in the fraction of LD-C-S-H phases, shown in Fig. 3(d).

\section{Creep response in light of cement's porous microstructure}

Final indirect confirmation for the scenario described above comes from the investigation of the pore size distribution of cement hydrates. From the mechanical results, we
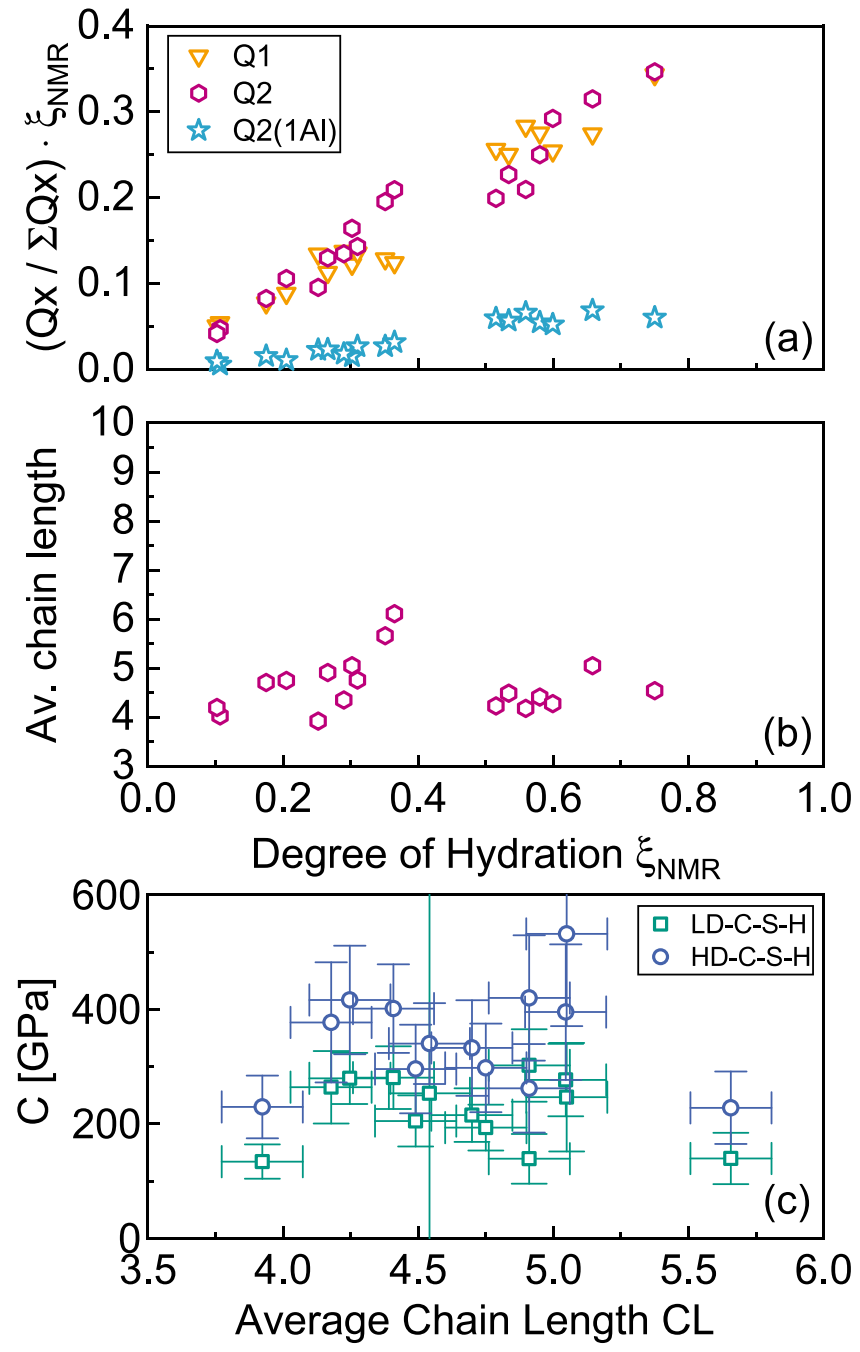

FIG. 5. (a) Fraction of C-S-H dimers and silicate end groups (designated $Q^{1}$ ) and polymeric units of silicate tetrahedra $\left(Q^{2}\right)$, including sites bound to tetrahedral $\mathrm{Al}$ in the chains $\left[Q^{2}(1 \mathrm{Al})\right]$, relative to the total amount of C-S-H phases formed multiplied by the degree of hydration $\xi_{\mathrm{NMR}}$ and plotted as a function of $\xi_{\mathrm{NMR}}$. (b) Average (Av.) aluminosilicate chain lengths (CLs) of the C-S-H polymers (see Appendix D for calculations) as a function of $\xi_{\mathrm{NMR}}$. The average standard deviation is estimated to be \pm 0.15 for the chain length values. (c) Creep modulus $C$ vs average aluminosilicate chain length of the C-S-H polymers, for both LD- and HD-C-S-H data.

compute the contribution of the LD- and HD-C-S-H phases' porosity to the overall porosity of the cement paste at various degrees of hydration. The porosity $p_{i}$ of the phase $i$ is calculated using $p_{i}=f_{i}\left(1-\eta_{i}\right)$, where $f_{i}$ designates the volume fraction [Fig. 3(d)] and $\eta_{i}$ designates the packing density of the phase $i$ [Fig. 3(h)]. As can be seen in Fig. 6 (green pentagons), the joint contribution of LD- and HD-C-S$\mathrm{H}$ phases to the total porosity of the sample is quasiconstant, independent of the degrees of hydration, with a mean value of 0.28 , which coincides remarkably well with the value of 0.27 predicted by Powers in his seminal work [67]. These estimates are compared with direct measurements of the porosity associated with pores of radius smaller than $20 \mathrm{~nm}$ and determined by nitrogen adsorption at $77 \mathrm{~K}$ (see Appendix D for 


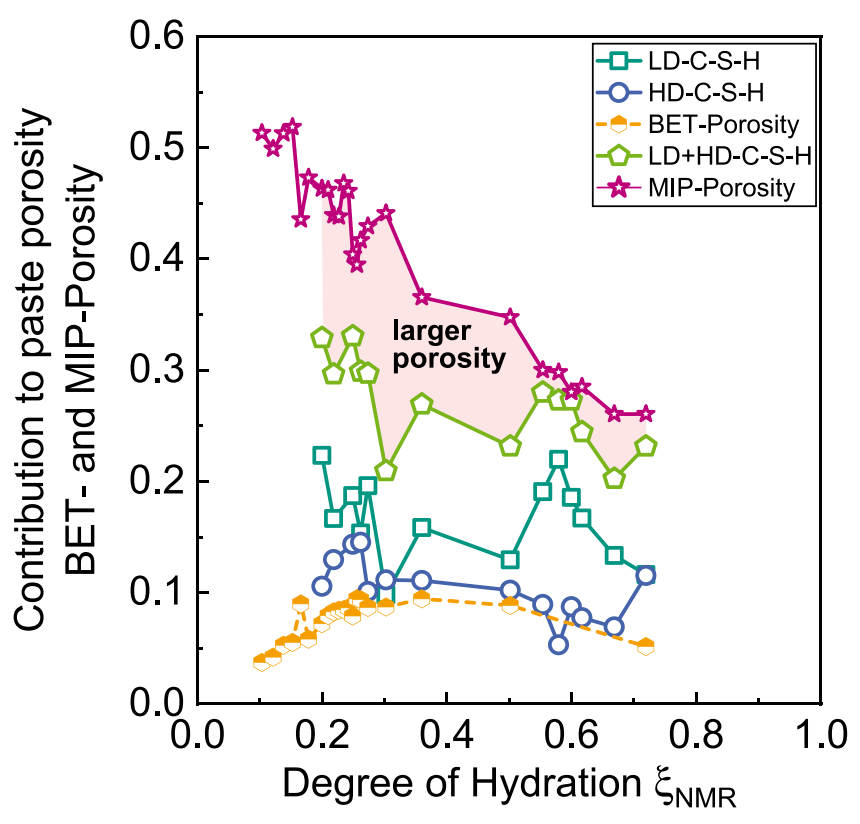

FIG. 6. Contribution to the cement paste total porosity of LDC-S-H, HD-C-S-H, and both phases as determined by statistical nanoindentation as a function of the degree of hydration $\xi_{\mathrm{NMR}}$. Results are compared with direct porosity measurements performed by nitrogen adsorption at $77 \mathrm{~K}$ and mercury intrusion porosimetry (MIP). BET stands for Brunauer, Emmett, and Teller.

details). The porosity measured by adsorption as a function of the degree of hydration shows a bell-shaped curve with a maximum at $\xi_{\mathrm{NMR}} \simeq 0.4$ (half-filled yellow circles in Fig. 6), which points toward a change in the hydration process for high degrees of hydration similar to what has been found by both nanoindentation testing and ${ }^{29} \mathrm{Si}$ NMR. This change has been associated with a transition from a free nucleation and growth process of C-S-H particles to a diffusion-controlled process [68]. A similar change is visible in the volume fraction of LD- and HD-C-S-H estimated from the mechanical tests at $\xi_{\mathrm{NMR}} \simeq 0.4$ [Fig. 3(d)]. Indeed, for $\xi_{\mathrm{NMR}} \lesssim 0.55$, the space provided by the larger pores is sufficient for the formation of both new LD- and HD-C-S-H, which corresponds to a steady increase in the specific surface area. In contrast, for $\xi_{\mathrm{NMR}} \gtrsim$ 0.55 , the structure formation is more and more dominated by compaction processes of the $\mathrm{C}-\mathrm{S}-\mathrm{H}$ nanoparticles, leading to a reduction of HD-C-S-H porosity. Note that this transition also corresponds to the sudden increase in the $Q^{1}$ vs $Q^{2}$ fraction, as observed in Fig. 5.

Finally, the larger-scale porosity of the samples was determined using mercury intrusion porosimetry (MIP), which gives access to the porosity in the range between approximately $5 \mathrm{~nm}$ and $500 \mu \mathrm{m}$ [69]. The difference between the porosity determined by MIP (purple stars in Fig. 6), i.e., the total pore volume, and the pore volume contribution from LDand HD-C-S-H phases can be interpreted as the capillary pore volume, i.e., the pores greater than approximately $30 \mathrm{~nm}$ in radius $[67,70]$. The results detailed in Fig. 6 clearly prove that for increasing degree of hydration, new C-S-H-gel is formed in the larger pores, reducing the overall porosity of the sample. The total volume-fraction increase of LD- and HD-C-SH phases and the corresponding contribution to porosity appear

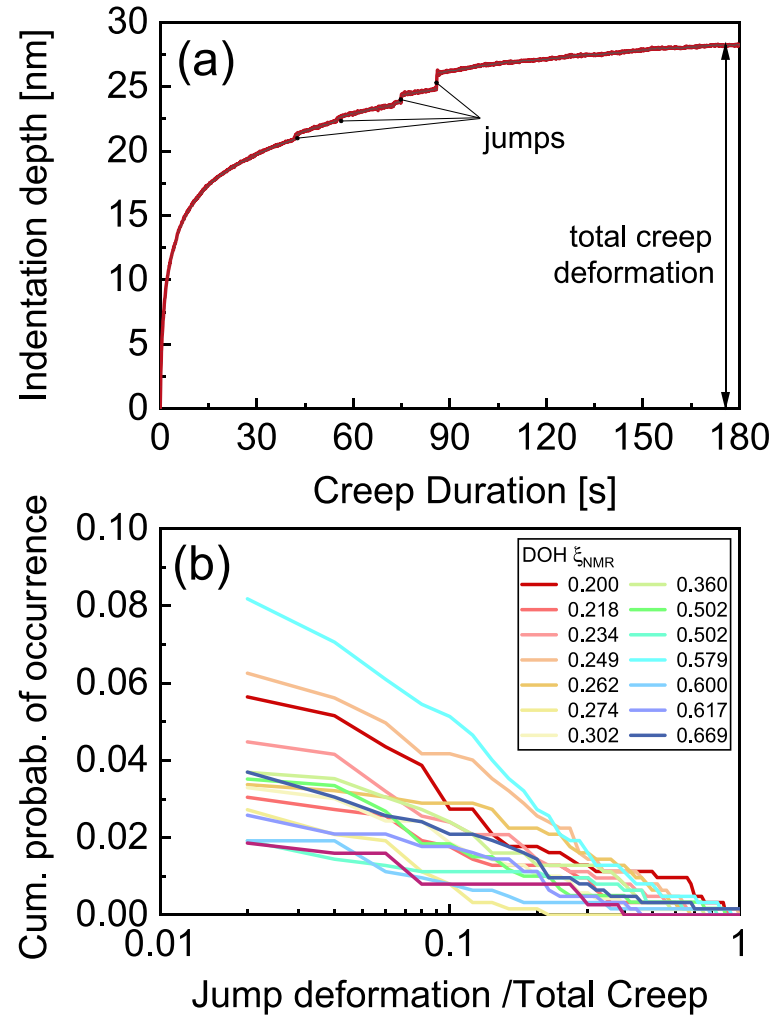

FIG. 7. (a) Example of indentation depth-vs-time curve afflicted with discontinuities (jumps). (b) Cumulative probability (Cum. probab.) of discontinuities and their contribution to the total creep deformation during the indentation creep phase for the investigated degrees of hydration (DOH) $\xi_{\mathrm{NMR}}$..

to be compensated by the increase in packing density of those phases. In agreement with the reasoning of Powers based on macroscopic experiments [67], these microstructural results show that the overall contribution of the C-S-H gel to the porosity remains roughly constant. This observation is linked to the continuous compaction of the system, to the increasing lack of free space for C-S-H formation, and especially to the fact that the C-S-H particles have a distinct size, posing an upper bound for compaction. More detailed research on the exact mechanisms is, however, necessary.

\section{Contribution of crack formation to the overall creep response}

In this section, we discuss the outliers detected in the indentation tests, i.e., the force-depth curves in which the creep phase shows irregularities such as a discontinuous behavior or jumps as illustrated in Fig. 7(a), and that may be interpreted as a microcrack. Concerning the question of whether and to what extent creep results from an irreversible microcracking proposed by Wittmann [39] and Bazant et al. [43], these "irregular" curves do contain important information. For each creep curve containing jumps, the total jump deformation was summed up from the individual jumps and compared with the total creep deformation (including jumps). Hereby, only jumps with an individual minimum jump height of $1 \mathrm{~nm}$ were considered, corresponding to three times the standard deviation of the measurement noise. The cumulative probability 

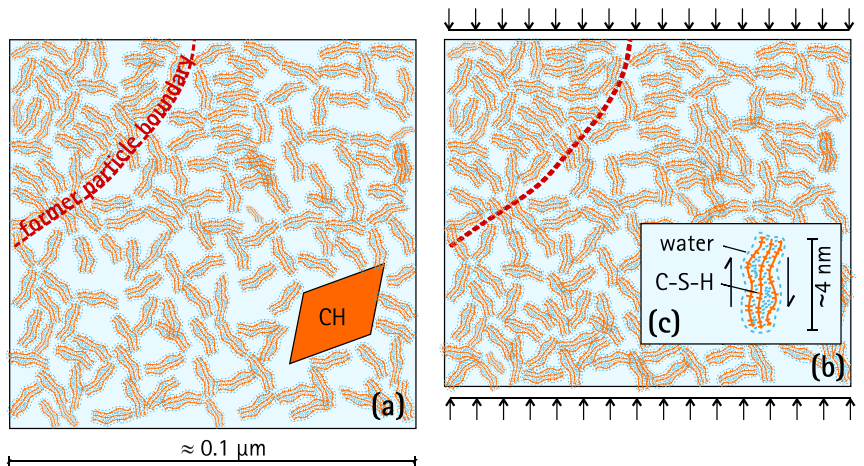

FIG. 8. Sketch of the microstructure of hardened cement paste in (a) the unloaded stage and (b) the loaded stage. Different areas of the sample show different packing densities (LD- and HD-C-S-H) associated with the spaces formerly occupied by particles prior to their dissolution (see dashed red lines). Deformation of the individual C-S-H particles [see inset (c)] subjected to loading as proven in molecular dynamics simulations might facilitate reorganization of the densely packed system and thus allow creep.

of jumps and their contribution to the total creep deformation is reported in Fig. 7(b) for various degrees of hydration. Only approximately 3-8\% of all indentation creep-vs-time curves showed discontinuities or jumps. In case jumps do occur, they can contribute significantly to the measured creep deformation-however, with the likelihood of large jumps occurring decreasing strongly. Moreover, no clear influence of the degree of hydration $\xi_{\text {NMR }}$ on the jump height was visible.

Coming back to the question of whether cement paste creep is significantly controlled by abrupt microcracking processes, our data indicate that this process eventually takes place but is negligible compared with other processes. This conclusion, however, is subject to the restriction that jumps in depth during the creep phase can also result from external mechanical vibrations. An analysis was carried out investigating the likelihood of a jump occurring as a function of the time of day-and thus of the likelihood of external vibrations in the building. We found that jumps occur during both daytime and nighttime but that the likelihood of jumps strongly increases during the daytime. This finding thus indicates that the contribution of microcracking to the total deformation is even lower than that suggested by Fig. 7(b).

\section{DISCUSSION AND CONCLUSION}

The present study provides strong experimental evidence that the creep response of hardening cement paste is primarily determined by the packing density of the C-S-H nanoparticles that compose the paste microstructure. We have shown that the creep modulus increases linearly for increasing packing density over the range $0.55 \leqslant \eta \leqslant 0.74$, or equivalently for decreasing distance between particles. This result suggests that the creep response of hardened cement paste mainly results from viscous sliding between $\mathrm{C}-\mathrm{S}-\mathrm{H}$ nanoparticles separated by nanoconfined water layers (see the sketch in Fig. 8). This microscopic picture of the creep response follows the same line of thought as the seminal considerations by Powers [37] and Wittmann [39], who suggested that the contribution to the total creep deformation, of a viscous deformation of individual C-S-H particles, is negligible. Moreover, our experimental findings strongly support the conclusions of recent molecular dynamics simulations of hydrated cement paste [48], in which the authors attribute the logarithmic creep response of cement paste (similar to the one we observe) to the sliding of $\mathrm{C}-\mathrm{S}-\mathrm{H}$ particles, provided that the water content is sufficient. In that context, our results allow ruling out the scenario following which creep in HCP results from a microcracking of the paste.

Finally, our results show that, in the limit of high degrees of hydration, the packing density of C-S-H particles has reached its maximum value. Such a jammed state of the hardened paste microstructure should either hinder creep deformation or lead to a dilation of the system under external stress. Yet, such effects are not observed. Therefore, for a strongly hydrated system, our results suggest that the viscoelastic responses of individual $\mathrm{C}-\mathrm{S}-\mathrm{H}$ particles eventually come into play to facilitate the interparticle sliding [20,71], and allow for the material to creep. This picture is compatible with numerical results reported in Refs. [47,48], in which C-S-H particles themselves can be subject to a creep deformation, which strongly facilitates the yielding process.

In conclusion, the combination of nanomechanical testing together with ${ }^{29} \mathrm{Si} \mathrm{NMR}$, and gas adsorption and mercury intrusion testing provides a comprehensive microscopic picture of the creep response of hydrating cement pastes over a large range of hydration degrees. Our results allow us to rank the three scenarios historically proposed to account for the creep deformation in cement paste. Beyond hydrating cement pastes, future work is needed to determine the extent to which our results can be applied to other reactive granular materials.

\section{ACKNOWLEDGMENTS}

This work was funded by a research stipend of the German Research Foundation (Deutsche Forschungsgemeinschaft, DFG) to M.H. (Reference No. HA 7917/1-1). The authors thank N. Chatterjee (MIT-EAS) for support in carrying out the WDS mappings as well as K. Ioannidou, S. Yip, P. Stemmermann, K. Garbev, and T. Petersen for extremely fruitful discussions.

\section{APPENDIX A: SAMPLE PREPARATION}

All cement paste samples were prepared by mixing ordinary Portland cement CEM I 42.5 R [65\% wt. tricalcium silicate $\left(\mathrm{Ca}_{3} \mathrm{SiO}_{5}\right) ; 15 \%$ wt. dicalcium silicate $\left(\mathrm{Ca}_{2} \mathrm{SiO}_{4}\right) ; 5 \%$ wt. tricalcium aluminate $\left(\mathrm{Ca}_{3} \mathrm{Al}_{2} \mathrm{O}_{6}\right) ; 2 \%$ wt. tetracalcium aluminate ferrite $\left.\left(\mathrm{Ca}_{4} \mathrm{Al}_{2} \mathrm{Fe}_{2} \mathrm{O}_{10}\right)\right]$ produced by Wittekind (Germany) with demineralized water, at a water-to-cement mass ratio $w / c=0.4$. Samples were cast in cylindrical tubes of length $50 \mathrm{~mm}$ and diameter $24 \mathrm{~mm}$ before being stored at $20{ }^{\circ} \mathrm{C}$ in a saturated lime $(\mathrm{CaO})$ solution. Following Ref. [56], the hydration reaction was stopped by solvent exchange with isopropanol at various points in time ranging from $7 \mathrm{~h}$ to 220 days after water addition to the dry cement. Once the hydration stopped, the cylindrical samples were then cut into disks, the surfaces of which were polished manually by using a series of $\mathrm{SiC}$ papers so as to reach a surface roughness of 
a few hundred nanometers [72]. Finally, the polished samples of hardened cement paste were dried in a thermal chamber at $60^{\circ} \mathrm{C}$ until they reached a constant mass. They were then tested mechanically by nanoindentation.

\section{APPENDIX B: STATISTICAL NANOINDENTATION}

The mechanical properties of the cement samples were characterized by nanoindentation (UNHT, Anton Paar). For each sample, $25 \times 25=625$ indents separated by $10 \mu \mathrm{m}$ were carried out on a square map using a three-sided, pyramidlike Berkovich indenter. Each indentation test consists in measuring the indentation depth of the indenter resulting from a symmetric load profile: a ramp of increasing load up to a constant value $P_{\max }$ maintained for $180 \mathrm{~s}$ so as to determine the creep response of the sample, followed by a ramp of decreasing load at a rate identical to the loading part. The maximum load $P_{\max }$ is chosen to yield indentation depths of approximately $300 \mathrm{~nm}$, probing the mechanical local properties of the hardened cement paste within a volume of about $1 \mu \mathrm{m}^{3}$. For each indent, the local values of the hardness $H$ and the indentation modulus $M$ were determined by the method of Oliver and Pharr [58], while the creep modulus $C$, which corresponds to the inverse of a creep rate, was determined following the method previously used by Vandamme and Ulm in Refs. [3,52] (also see Sec. IV). For a given sample, an indentation grid yields a set of 625 triplets $(H, M, C)$, which is analyzed with a Gaussian mixture modeling extensively described in Ref. [60]. Such analysis, combined with Bayesian information criteria, allows us to determine the most likely number of phases based on the Gaussian clustering of the mechanical properties and the chemical content at the locus of each indent (see following paragraph). The analysis yields between three and five phases, which correspond to the phases composing the hardened cement paste: low-density C-S-H (LD-C-S-H), high-density C-S-H (HD-C-S-H), calcium hydroxide $(\mathrm{CH})$, mixed phases, and unhydrated clinker.

\section{APPENDIX C: CHEMICAL SURFACE MAPPING}

The entire surface of the hardened cement paste sample is coated with a carbon layer of $20 \mathrm{~nm}$ thickness and dried in an ultravacuum $\left(8 \times 10^{-9}\right.$ bar $)$ for at least $24 \mathrm{~h}$. The chemical composition of the sample at the locus of the indentation grid is determined using wavelength-dispersive $\mathrm{x}$-ray spectroscopy (WDS) with a scanning electron microscope (SEM, JEOL JXA-8200). SEM backscatter images and WDS maps with dimensions of $368 \times 276 \mu \mathrm{m}$ (corresponding to $1024 \times$ 768 pixels) were acquired with a resolution of $0.36 \mu \mathrm{m}$. The beam voltage, current, working distance, and dwell time were set to $15 \mathrm{kV}, 10 \mathrm{nA}, 11 \mathrm{~mm}$, and $40 \mathrm{~ms}$ per spot, respectively. The WDS mapping was performed for the following species: calcium $(\mathrm{Ca})$, silicon $(\mathrm{Si})$, aluminum $(\mathrm{Al})$, iron $(\mathrm{Fe})$, sulfur $(\mathrm{S})$, magnesium $(\mathrm{Mg})$, sodium $(\mathrm{Na})$, and potassium $(\mathrm{K})$.

\section{APPENDIX D: BULK TESTING TECHNIQUES}

Single-pulse ${ }^{29} \mathrm{Si}$ MAS NMR spectra were acquired on a Bruker $400-\mathrm{MHz}(9.39 \mathrm{~T})$ spectrometer using a home-built cross-polarization MAS (CP/MAS) probe for 7-mm-outerdiameter rotors, a $45^{\circ}$ excitation pulse, a 30-s relaxation delay, and typically 2048 scans. The resulting spectra were deconvolved providing relative intensities for alite, belite, and the $Q^{1}, Q^{2}$, and $Q^{2}(1 \mathrm{Al})$ resonances of the C-S-H phase. From these intensities, the degrees of silicate hydration, $\xi_{\mathrm{NMR}}=$ $\left[I\left(Q^{1}\right)+I\left(Q^{2}\right)+I\left(Q^{2}(1 \mathrm{Al})\right)\right] / I_{\mathrm{tot}}$ (with $I_{\mathrm{tot}}$ designating the total signal intensity) and the average chain length of aluminosilicate tetrahedra, $\mathrm{CL}=2\left[Q^{1}+Q^{2}+3 / 2 Q^{2}(1 \mathrm{Al})\right] / Q^{1}$, were calculated (see Ref. [66]). Moreover, the porosity of the hardened cement paste was determined by mercury intrusion porosimetry (MIP) using a Micromeritics Autopore III 9420 instrument with $\mathrm{Hg}$ pressures up to $400 \mathrm{MPa}$ and by nitrogen adsorption at $77 \mathrm{~K}$ using a Micromeritics ASAP 2000 instrument. Here, the MIP method was used to measure the total porosity of the sample, with pores as small as approximately $5 \mathrm{~nm}$ in radius [69]. The pore size distribution accessed by nitrogen adsorption was derived using the Barrett-JoynerHalenda $(\mathrm{BJH})$ algorithm, covering pores with a diameter between $1 \mathrm{~nm}$ and approximately $120 \mathrm{~nm}$, thus encompassing both LD- and HD-C-S-H and parts of the capillary porosity (i.e., pores with a radius larger than $20 \mathrm{~nm}$ ) [69]. In order to identify the porosity and pore size distribution of the individual phases identified via the nanoindentation coupled to surface chemistry, a superposition of Gaussian functions was fitted to the pore-volume-vs-log-pore-radius distribution data obtained in BET testing thus identifying the pore size clusters present in the paste. At least three clusters could be identified with mean pore radii of 6,15 , and $28 \mathrm{~nm}$. Hereby, the identified mean pore radii of the first two clusters (i.e., 6 and $15 \mathrm{~nm}$ ) correspond well to the sizes derived from NMR relaxometry data on similar pastes (see Ref. [73]). The pore volume contribution of the first two clusters as shown in Fig. 6 (designated "BET-Porosity") was calculated by integrating the pore size distribution in the range between 1 and $20 \mathrm{~nm}$, where the upper threshold results from the mean pore radius of cluster 2 (i.e., $15 \mathrm{~nm}$ ) plus three times its standard deviation. These results suggest that BET pore clusters No. 1 and No. 2 (i.e., 6 and $15 \mathrm{~nm}$ ) can be attributed to HD-C-S-H, whereas the properties of LD-C-S-H are significantly affected by larger pores. It should be noted that this finding somewhat contradicts rough estimates on the pore radius of LD-C-S-H by Jennings [27], which, however, were not measured but calculated from estimates of density and specific surface area and thus are prone to various possible errors.
[1] W. Weibull, The Phenomenon of Rupture in Solids, edited by the Royal Swedish Institute for Engineering Research (Generalstabens Litografiska Anstalts Foörlag, Stockholm, 1939).

[2] V. B. Nguyen, T. Darnige, A. Bruand, and E. Clement, Creep and Fluidity of a Real Granular Packing near Jamming, Phys. Rev. Lett. 107, 138303 (2011).
[3] M. Vandamme and F.-J. Ulm, Nanogranular origin of concrete creep, Proc. Natl. Acad. Sci. USA 106, 10552 (2009).

[4] M.-C. Miguel, A. Vespignani, M. Zaiser, and S. Zapperi, Dislocation Jamming and Andrade Creep, Phys. Rev. Lett. 89, 165501 (2002). 
[5] B. Jakobsen, H. F. Poulsen, U. Lienert, J. Almer, S. D. Shastri, H. O. Sørensen, C. Gundlach, and W. Pantleon, Formation and subdivision of deformation structures during plastic deformation, Science 312, 889 (2006).

[6] F. F. Csikor, C. Motz, D. Weygand, M. Zaiser, and S. Zapperi, Dislocation avalanches, strain bursts, and the problem of plastic forming at the micrometer scale, Science 318, 251 (2011).

[7] M.-C. Miguel, L. Laurson, and M. J. Alava, Material yielding and irreversible deformation mediated by dislocation motion, Eur. Phys. J. B 64, 443 (2008).

[8] A. Vinogradov, I. S. Yasnokov, and Y. Estrin, Evolution of Fractal Structures in Dislocation Ensembles during Plastic Deformation, Phys. Rev. Lett. 108, 205504 (2012).

[9] T. Sentjabrskaja, P. Chaudhuri, M. Hermes, W. C. K. Poon, J. Horbach, S. U. Egelhaaf, and M. Laurati, Creep and flow of glasses: strain response linked to the spatial distribution of dynamical heterogeneities, Sci. Rep. 5, 11884 (2015).

[10] P. Cao, M. P. Short, and S. Yip, Understanding the mechanisms of amorphous creep through molecular simulation, Proc. Natl. Acad. Sci. USA 114, 13631 (2017).

[11] S. Aime, L. Ramos, and L. Cipelletti, Microscopic dynamics and failure precursors of a gel under mechanical load, Proc. Natl. Acad. Sci. USA 115, 3587 (2018).

[12] C. Liu, K. Martens, and J.-L. Barrat, Mean-Field Scenario for the Athermal Creep Dynamics of Yield-Stress Fluids, Phys. Rev. Lett. 120, 028004 (2018).

[13] L. Cipelletti, K. Martens, and L. Ramos, Microscopic precursors of failure in soft matter, Soft Matter 16, 82 (2020).

[14] M. Siebenbürger, M. Ballauf, and T. Voigtmann, Creep in Colloidal Glasses, Phys. Rev. Lett. 108, 255701 (2012).

[15] M. Leocmach, C. Perge, T. Divoux, and S. Manneville, Creep and Fracture of a Protein Gel under Stress, Phys. Rev. Lett. 113, 038303 (2014).

[16] M. R. Kuhn and J. K. Mitchell, New perspectives on soil creep, J. Geotech. Eng. 119, 507 (1993).

[17] E. T. Bowman and K. Soga, Creep, ageing and microstructural change in dense granular materials, Soils Found. 43, 107 (2003).

[18] O. Reynolds, On the dilatancy of media composed of rigid particles in contact. with experimental illustrations, London Edinburgh Philos. Mag. J. Sci. 20, 469 (1885).

[19] R. P. Behringer and B. Chakraborty, The physics of jamming for granular materials: A review, Rep. Prog. Phys. 82, 012601 (2019).

[20] G. R. McDowell, Micromechanics of creep of granular materials, Géotechnique 53, 915 (2003).

[21] H. Shi, Deformation of cohesive granular materials: Micro influences macro, Ph.D. thesis, University of Twente, Enschede, Netherlands, 2019.

[22] R. Raj and P. E. D. Morgan, Activation energies for densification, creep, and grain-boundary sliding in nitrogen ceramics, J. Am. Ceram. Soc. 64, C-143 (1981).

[23] S. de Meer and C. J. Spiers, Uniaxial compaction creep of wet gypsum aggregates, J. Geophys. Res. Solid Earth 102, 875 (1997).

[24] C. J. Spiers, P. M. T. M. Schutjens, R. H. Brzesowsky, C. J. Peach, J. L. Liezenberg, and H. J. Zwart, Experimental determination of constitutive parameters governing creep of rocksalt by pressure solution, Geol. Soc. Spec. Publ. 54, 215 (1990).
[25] D. K. Dysthe, Y. Podladchikov, F. Renard, J. Feder, and B. Jamtveit, Universal Scaling in Transient Creep, Phys. Rev. Lett. 89, 246102 (2002)

[26] H. S. Müller and M. Haist, Concrete, in Structural Concrete: Textbook on Behaviour, Design and Performance, fib Bulletin 51, 2nd ed. (Fédération internationale du béton, Lausanne, 2009), Vol. 1, Chap. 3.1, pp. 35-149.

[27] H. M. Jennings, A model for the microstructure of calcium silicate hydrate in cement paste, Cem. Concr. Res. 30, 101 (2000).

[28] J. W. Bullard, H. M. Jennings, R. A. Livingston, A. Nonat, G. W. Scherer, J. S. Schweitzer, K. L. Scrivener, and J. J. Thomas, Mechanisms of cement hydration, Cem. Concr. Res. 41, 1208 (2011).

[29] R. J.-M. Pellenq, J. Caillol, and A. Delville, Electrostatic attraction between two charged surfaces: A $(N, V, T)$ Monte Carlo simulation, J. Phys. Chem. B 101, 8584 (1997).

[30] R. J.-M. Pellenq and H. Van Damme, Why does concrete set?: The nature of cohesion forces in hardened cement-based materials, MRS Bull. 29, 319 (2004).

[31] C. Plassard, E. Lesniewska, I. Pochard, and A. Nonat, Nanoscale experimental investigation of particle interactions at the origin of the cohesion of cement, Langmuir 21, 7263 (2005).

[32] R. J.-M. Pellenq, N. Lequeux, and H. van Damme, Engineering the bonding scheme in C-S-H: The iono-covalent framework, Cem. Concr. Res. 38, 159 (2008).

[33] K. Ioannidou, M. Kanduc, L. Li, D. Frenkel, J. Dobnikar, and E. Del Gado, The crucial effect of early-stage gelation on the mechanical properties of cement hydrates, Nat. Commun. 7, 12106 (2016).

[34] F. H. Wittman, Interaction of hardened cement paste and water, J. Am. Ceram. Soc. 56, 409 (1973).

[35] L. J. Parrott, An examination of the effects of age at loading upon the creep of hardened cement paste, Mag. Concr. Res. 25, 197 (1973).

[36] B. T. Tamtsia and J. J. Beaudoin, Basic creep of hardened cement paste a re-examination of the role of water, Cem. Concr. Res. 30, 1465 (2000).

[37] T. C. Powers, The thermodynamics of volume change and creep, Mater. Constr. (Paris) 1, 487 (1968).

[38] R. F. Feldman, Mechanism of creep of hydrated Portland cement paste, Cem. Concr. Res. 2, 521 (1972).

[39] F. H. Wittmann, Creep and shrinkage mechanisms, in Creep and Shrinkage in Concrete Structures, edited by Z. P. Bazant and F. H. Wittmann (Wiley, New York, 1982), pp. 129-162.

[40] N. H. Brown and B. B. Hope, The creep of hydrated cement paste, Cem. Concr. Res. 6, 475 (1976).

[41] A. Bentur, R. L. Berger, F. V. Lawrence, Jr., N. B. Milestone, S. Mindess, and J. F. Young, Creep and drying shrinkage of calcium silicate pastes III. A hypothesis of irreversible strains, Cem. Concr. Res. 9, 83 (1979).

[42] Z. P. Bazant and S. Prasannan, Solidification theory for concrete creep I: Formulation, J. Eng. Mech. 115, 1691 (1989).

[43] Z. P. Bazant, A. B. Hauggaard, S. Baweja, and F.-J. Ulm, Microprestress-solidification theory for concrete creep. I: Aging and drying effects, J. Eng. Mech. 123, 1188 (1997).

[44] H. M. Jennings, B. J. Dalgleish, and P. L. Pratt, Morphological development of hydrating tricalcium silicate as examined by electron microscopy techniques, J. Am. Ceram. Soc. 64, 567 (1981). 
[45] S. Diamond and D. Bonen, Microstructure of hardened cement paste-A new interpretation, J. Am. Ceram. Soc. 76, 2993 (1993).

[46] K. Ioannidou, K. J. Krakowiak, M. Bauchy, C. G. Hoover, E. Masoero, S. Yip, F.-J. Ulm, P. Levitz, R. J.-M. Pellenq, and E. Del Gado, Mesoscale texture of cement hydrates, Proc. Natl. Acad. Sci. USA 113, 2029 (2016).

[47] H. Manzano, E. Masoero, I. Lopez-Arbeloa, and H. M. Jennings, Shear deformations in calcium silicate hydrates, Soft Matter 9, 7333 (2013).

[48] A. Morshedifard, S. Masoumi, and M. J. Abdolhosseini Qomi, Nanoscale origins of creep in calcium silicate hydrates, Nat. Commun. 9, 1785 (2018).

[49] H. M. Jennings, Refinements to colloidal models of C-S-H in cement, Cem. Concr. Res. 38, 275 (2008).

[50] P. D. Tennis and H. M. Jennings, A model for two types of calcium silicate hydrate in the microstructure of Portland cement pastes, Cem. Concr. Res. 30, 855 (2000).

[51] T. C. Powers and T. L. Brownyard, Studies of the Physical Properties of Hardened Portland Cement Paste, Bulletin 22 (Research Laboratories of the Portland Cement Association, Chicago, 1948).

[52] M. Vandamme and F.-J. Ulm, Nanoindentation investigation of creep properties of calcium silicate hydrates, Cem. Concr. Res. 52, 38 (2013).

[53] J. F. Young, The Microstructure of Hardened Portland Cement Paste. Creep and Shrinkage in Concrete Structures (Wiley, New York, 1982).

[54] T. Zhou, K. Ioannidou, F.-J. Ulm, M. Z. Bazant, and R. J.M. Pellenq, Multiscale poromechanics of wet cement paste, Proc. Natl. Acad. Sci. USA 116, 10652 (2019).

[55] M. J. Abdolhosseini Qomi, K. J. Krakowiak, M. Bauchy, K. L. Stewart, R. Shahsavari, D. Jagannathan, D. B. Brommer, A. Baronnet, M. J. Buehler, S. Yip, F.-J. Ulm, K. J. Van Vliet, and R. J.-M. Pellenq, Combinatorial molecular optimization of cement hydrates. Nat. Commun. 5, 4960 (2014).

[56] J. Zhang and G. W. Scherer, Comparison of methods for arresting hydration of cement, Cem. Concr. Res. 41, 1024 (2011).

[57] W. Oliver and G. Pharr, An improved technique for determining hardness and elastic modulus using load and displacement sensing indentation experiments, J. Mater. Res. 7, 1564 (1992).

[58] W. C. Oliver and G. M. Pharr, Measurement of the hardness and elastic modulus by instrumented indentation: Advances in understanding and refinements to methodology, J. Mater. Res. 19, 3 (2004).

[59] M. Vandamme, C. A. Tweedie, G. Constantinides, F.-J. Ulm, and K. J. Van Vliet, Quantifying plasticity-independent creep compliance and relaxation of viscoelastoplastic materials under contact loading, J. Mater. Res. 27, 302 (2012).

[60] K. J. Krakowiak, W. Wilson, S. Musso, S. James, and F.-J. Ulm, Inference of the phase-to-mechanical property link via coupled $\mathrm{X}$-ray spectrometry and indentation analysis: Application to cement-based materials, Cem. Concr. Res. 67, 271 (2015).

[61] The influence of aluminate phases could be neglected due to the low aluminate content of the investigated cement.

[62] L. Sorelli, G. Constantinides, F.-J. Ulm, and F. Toutlemonde, The nano-mechanical signature of ultra high performance concrete by statistical nanoindentation techniques, Cem. Concr. Res. 38, 1447 (2008).

[63] F.-J. Ulm, M. Vandamme, C. Bobko, J. A. Ortega, K. Tai, and C. Ortiz, Statistical indentation techniques for hydrated nanocomposites: Concrete, bone, and shale, J. Am. Ceram. Soc. 90, 2677 (2007).

[64] S. Cariou, F.-J. Ulm, and L. Dormieux, Hardness and packing density scaling relations for cohesive-frictional porous materials, J. Mech. Phys. Solids 56, 924 (2008).

[65] M. Abuhaikal, K. Ioannidou, T. Petersen, R. J.-M. Pellenq, and F.-J. Ulm, Le châtelier's conjecture: Measurement of colloidal eigenstresses in chemically reactive materials, J. Mech. Phys. Solids 112, 334 (2018).

[66] M. D. Andersen, H. J. Jakobsen, and J. Skibsted, Incorporation of aluminum in the calcium silicate hydrate $(\mathrm{C}-\mathrm{S}-\mathrm{H})$ of hydrated Portland cements: A high-field ${ }^{27} \mathrm{Al}$ and ${ }^{29} \mathrm{Si}$ MAS NMR investigation, Inorg. Chem. 42, 2280 (2003).

[67] T. C. Powers, The non-evaporable water content of hardened Portland-cement paste-Its significance for concrete research and its method of determination, ASTM Bull. 158, 68 (1949).

[68] S. A. Fitzgerald, D.A. Neumann, J. J. Rush, D. P. Bentz, and R. A. Livingston, In situ quasi-elastic neutron scattering study of the hydration of tricalcium silicate, Chem. Mater. 10, 397 (1998).

[69] K. K. Aligizaki, Pore Structure of Cement-Based Materials. Testing, Interpretation and Requirements (Taylor \& Francis, New York, 2006).

[70] S. Mindess, F. Young, and D. Darwin, Concrete (Prentice-Hall, Upper Saddle River, NJ, 2003).

[71] M. E. Cates, J. P. Wittmer, J.-P. Bouchaud, and P. Claudin, Jamming, Force Chains, and Fragile Matter, Phys. Rev. Lett. 81, 1841 (1998).

[72] M. Miller, C. Bobko, M. Vandamme, and F.-J. Ulm, Surface roughness criteria for cement paste nanoindentation, Cem. Concr. Res. 38, 467 (2008).

[73] W. P. Halperin, J.-Y. Jehng, and Y.-Q. Song, Application of spin-spin relaxation to measurement of surface area and pore size distributions in a hydrating cement paste, Magn. Reson. Imaging 12, 169 (1994). 